\title{
ASYMMETRIC STOKES Q, U AND V LINE PROFILES OB- SERVED IN SUNSPOTS
}

\author{
J. SÁNCHEZ ALMEIDA \\ Instituto de Astrofísica de Canarias, 38200 La Laguna, Tenerife, Spain \\ B.W. LITES \\ High Altitude Observatory, National Center for Atmospheric Research, \\ Boulder, Colorado 80307-3000, USA
}

\begin{abstract}
We present a systematic description of the asymmetric shapes the Stokes line profiles exhibit in sunspots. These asymmetries seem to be caused by gradients of both magnetic field and velocity in the photospheres of sunspots.
\end{abstract}

\section{INTRODUCTION}

Although asymmetric Stokes Q, U and V line profiles have been observed in sunspots since the sixties (e.g., Kjeldseth-Moe 1967), no systematic study of the spatial distribution of shapes, magnitudes etc. exits in the literature. The present work describes a first attempt to give a systematic characterization of the asymmetries of these Stokes profiles. We also outline a fist order interpretation of the underlying physical cause.

\section{SPATIAL VARIATION}

We have analyzed Stokes $\mathrm{Q}, \mathrm{U}$ and $\mathrm{V}$ profiles gathered in four solar regions $\left(125^{\prime \prime} \times 125^{\prime \prime}\right)$, each one containing a large, roughly symmetric, sunspot (see Lites \& Skumanich 1990 for details). The basic observational parameters of these data are:

- Spectral line: Fel 630.25 nm.

- Telescope and polarimeter: the coronograph at Sacramento Peak together with the Stokes II HAO polarimeter (Baur et al. 1981).

- Spectral resolution: $\sim 10 \mathrm{~m} \AA /$ pix smoothed to $\sim 35 \mathrm{~m} \AA$.

- Angular resolution: pixel size as well as sampling interval $\sim 5^{\prime \prime}$.

- Instrumental polarization: negligible (see Sánchez Almeida \& Lites 1992 for details).

One can divide each sunspot into three zones according to the type of asymmetry the corresponding Stokes profiles present there: the center-side penumbra (penumbra closest to the center of the disk), the umbra and the limb-side penumbra. Line shapes vary smoothly from one zone to another, though. Center-side 
penumbral profiles show blue lobes broader and generally lower in amplitude than the red lobes. Red lobes turn out to be broader in the limb-side penumba. Finally, asymmetries become less pronounced in the umbral profiles. Fig. 1 shows various representative Stokes V profiles.

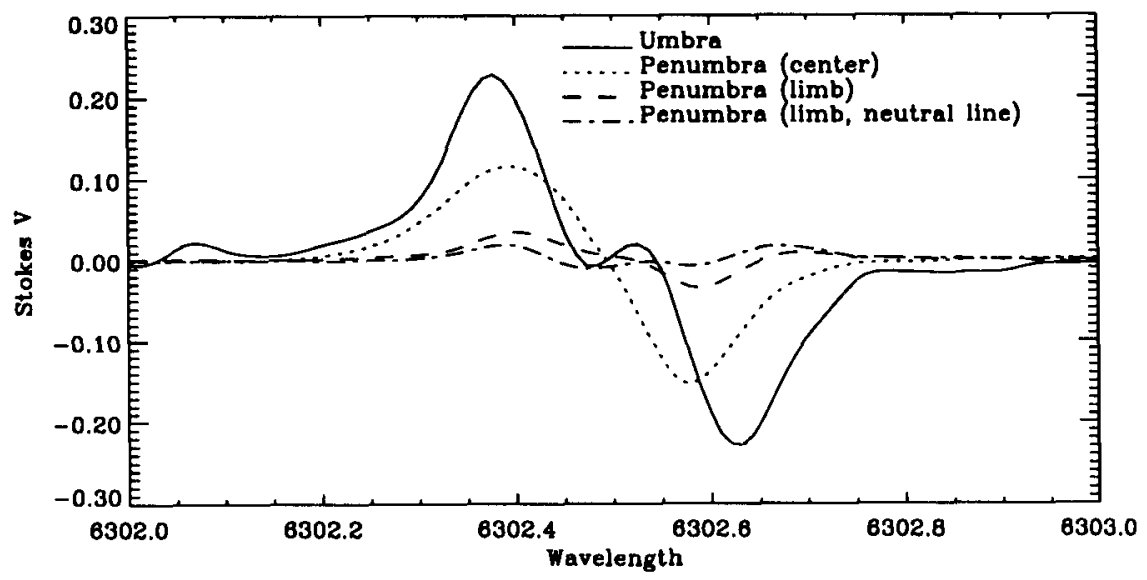

Fig. 1. Various typical examples of the shapes the Stokes V profiles (circular polarization versus wavelength in $\AA$ ) present in different parts of the sunspot.

\section{GRADIENTS OF MAGNETIC FIELD AND VELOCITY}

We tried to reproduce the observed asymmetries invoking line-of-sight gradients of both magnetic field and velocity. Spatial inhomogeneities smaller than our resolution element might also cause the asymmetries of the Stokes profiles; nevertheless, they cannot produce the broad-band circular polarization sunspots generate (Makita \& Ohki 1986 and references therein).

Applying the inversion technique by Landolfi (1983), we find that an Evershed flow whose magnitude increases with depth roughly reproduces the observed asymmetric profiles. Such gradient fits within the classical picture of the Evershed flow. In order to reproduce the observed broad-band circular polarization quoted above, the magnetic field lines have to become more horizontal as the line-of-sight goes deeper into the penumbral atmosphere (see Sánchez Almeida \& Lites 1992 for details).

\section{CONCLUSIONS}

The asymmetric shapes of the Stokes $Q, U$ and V line profiles of FeI 630.25 $\mathrm{nm}$ vary systematically across sunspots: from the center-side penumbra through the umbra to the limb-side penumbra. Such variation seems to be due to the Evershed flow whose magnitude increases with depth. In addition, the basic observational properties of the broad-band circular polarization are reproduced 
if, on top of this variation of the Evershed flow, one assumes that the penumbral magnetic field lines become more horizontal as the optical depth increases.

\section{ACKNOWLEDGEMENTS}

A detailed discussion on the techniques and results presented at this colloquium will appear in the paper by Sánchez Almeida \& Lites (1992).

The work was partly funded by the DGICYT under project PB91-0530.

The NCAR is sponsored by the National Science Foundation.

\section{REFERENCES}

Baur, T.G., Elmore, D.E., Lee, R.H., Querfeld, C.W., \& Rogers, S.R. 1981, Solar Phys. 70, 395

Kjeldseth-Moe, O. 1967, in IAU Symp. 35 Structure and development of solar active regions, ed K.O. Kiepenheuer, Reidel, p. 202

Landolf, M 1987, Solar Phys. 109, 287

Lites, B.W., \& Skumanich, A. 1990, Ap. J., 348, 747

Makita, M., \& Ohki, Y. 1986, Ann. Tokyo Astron. Obs. 21, 1

Sánchez Almeida, J., \& Lites B.W. 1992, Ap. J., 398, in press 